\title{
On Some Banhatti Indices of Triangular Silicate, Triangular Oxide, Rhombus Silicate and Rhombus Oxide Networks
}

\author{
Fazal Dayan ${ }^{1,}$, , Muhammad Javaid ${ }^{1}$, Umar Ali $^{1}$, Bilal Ahmad ${ }^{2}$, Muhammad Zulqarnain ${ }^{1}$ \\ ${ }^{1}$ Department of Mathematics, School of Science, University of Management and Technology, Lahore, Pakistan \\ ${ }^{2}$ Department of Mathematics and Statistics, The University of Lahore, Lahore, Pakistan
}

Email address:

fazaldayan1@gmail.com (F. Dayan)

${ }^{*}$ Corresponding author

\section{To cite this article:}

Fazal Dayan, Muhammad Javaid, Umar Ali, Bilal Ahmad, Muhammad Zulqarnain. On Some Banhatti Indices of Triangular Silicate, Triangular Oxide, Rhombus Silicate and Rhombus Oxide Networks. American Journal of Information Science and Technology. Vol. 2, No. 2, 2018, pp. 42-49. doi: 10.11648/j.ajist.20180202.13

Received: April 13,2018; Accepted: May 2, 2018; Published: June 4, 2018

\begin{abstract}
Silicates are the largest, the most complicated and the most interesting class of minerals by far. Kulli introduced the Banhatti indices of a graph. In this paper the general K-Banhatti indices, first and second K-Banhatti indices, K hyper Banhatti indices and modified $\mathrm{K}$ Banhatti indices for triangular silicate network, triangular oxide network, rhombus oxide network and rhombus silicate networks are computed.
\end{abstract}

Keywords: Triangular Silicate Network, Triangular Oxide Network, Rhombus Oxide Network, Rhombus Silicate Network, Banhatti Indices

\section{Introduction}

Kulli introduced the first and second K-Banhatti indices of a graph in [1]. These are defined as,

$$
B_{1}(G)=\sum_{u e}\left[d_{G}(u)+d_{G}(e)\right]
$$

and

$$
B_{2}(G)=\sum_{u e}\left[d_{G}(u) d_{G}(e)\right]
$$

where $u e$ indicate that the vertex $u$ and edge $e$ are incident in $G$. Kulli also defined some properties of these newly defined indices. The coindices of K-Banhatti indices were also defined in his work. Later kulli defined $\mathrm{K}$ hyper-Banhatti indices of $\mathrm{V}$ Phenylenic nanotubes and nanotorus [2]. Gutman et al developed relations between Banhatti and Zagreb indices and discussed the lower and upper bounds for Banhatti indices of a connected graph in terms of Zagreb indices [3]. Kulli et al also computed Banhatti indices for certain families of benzenoid systems [4]. Moreover, Kulli introduced multiplicative hyperBanhatti indices and coindices, Banhatti geometric-arithmetic index connectivity Banhatti indices for certain families of benzenoid systems [5-7].

Silicates are the largest, the most complicated and the most interesting class of minerals by far. $\mathrm{SiO}_{4}$ tetrahedron is the basic chemical unit of silicates. The silicates sheets are rings of tetrahedrons linked by shared oxygen nodes to other rings in two dimensional planes producing a sheet like structures. A silicate can be obtained by fusing a metal oxide or a metal carbonate with sand. Essentially every silicate contains $\mathrm{SiO}_{4}$ tetrahedron. The corner and the center vertices represent oxygen and silicon ions respectively. These vertices are called oxygen nodes and silicon node respectively. A Silicate network is obtained in different ways. Paul Manuel has constructed a silicate network from a honeycomb network. In Figure $1, \mathrm{SiO}_{4}$ tetrahedra is shown where the corner and the center vertices represent oxygen and silicon ions respectively.

Computing topological indices in mathematical chemistry is an important branch. Topological index has become a very useful tool in the prediction of physio chemical and pharmacological properties of a compound.

The number of vertices and edges are the topological index molecular structure matters. The main ingredients of the molecular topological models are topological indices which are the topological characterization of molecules by means of numerical invariants. These models are instrumental in the discovery of new applications of molecules with specific chemical, pharmacological and biological properties. The first use of a topological index was made by the chemist Harold Wiener in 1947 [8]. These indices are used by various 
researchers in their studies. In recent years many researchers have worked on computing topological indices [9-17].

The general first and second K-Banhatti indices of a graph are defined as [7]:

$$
\begin{aligned}
& B_{1}{ }^{a}(G)=\sum_{u e}\left[d_{G}(u)+d_{G}(e)\right]^{a} \\
& B_{2}{ }^{a}(G)=\sum_{u e}\left[d_{G}(u) d_{G}(e)\right]^{a},
\end{aligned}
$$

where $a \in \mathbb{R}$.

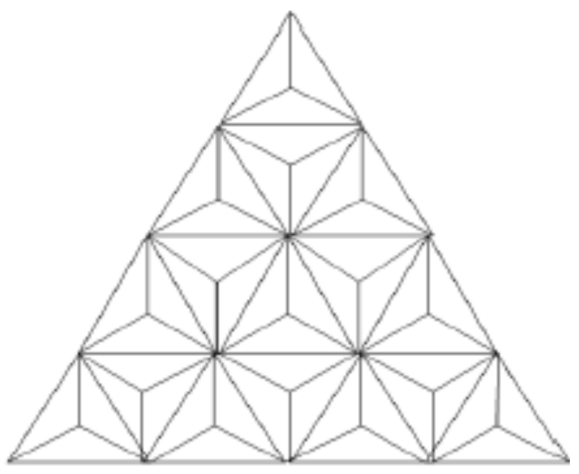

Figure 1. Triangular silicate network of order 4.

\section{Main Results}

\subsection{Calculating Banhatti Indices for Silicate Network $\operatorname{Ts} L(n)$}

A silicate network $\operatorname{Ts} L(n)$ of dimension 4 is given in figure 1 . The number of vertices and the number of edges in a triangular oxide network are given by

$$
\begin{gathered}
|V(\operatorname{TsL}(n))|=\left[\frac{(n-1)(n-2)}{2}\right] 3(n-1)+n^{2}+3 \\
|E(\operatorname{TsL}(n))|=\frac{3}{2} n(n+1)
\end{gathered}
$$

In a triangular oxide network, from level 4 there are 6 types of edges based on the degree of the vertices of each edge. In table 1 the edge degree partition of triangular silicate network $\operatorname{Ts} L(n)$ of order 4 is shown.

Table 1. Edge degree partition of triangular silicate network TsL(n) of order 4.

\begin{tabular}{lll}
\hline $\boldsymbol{d}_{\boldsymbol{G}}(\boldsymbol{u}), \boldsymbol{d}_{\boldsymbol{G}}(\boldsymbol{e}) \backslash \boldsymbol{e}=\boldsymbol{u} \boldsymbol{v} \in \boldsymbol{E}(\boldsymbol{G})$ & $\boldsymbol{d}_{\boldsymbol{G}}(\boldsymbol{e})$ & Total number of edges \\
\hline$(3,3)$ & 4 & 3 \\
$(3,7)$ & 8 & $33+9(n-4)$ \\
$(7,7)$ & 12 & $3(n-1)$ \\
$(3,12)$ & 13 & $6 \frac{(n-2)(n-1)}{2}$ \\
$(7,12)$ & 17 & $12+6(n-4)$ \\
$(12,12) 1$ & 22 & $3 \frac{(n-3)(n-2)}{2} 1$ \\
\hline
\end{tabular}

Theorem: For a triangular silicate network, $\operatorname{Ts} L(n)$, the general first and second K-Banhatti indices are given by

$$
\begin{gathered}
B_{1}{ }^{a}(\operatorname{Ts} L(n))=6 \times 7^{a}+(9 n-3)\left[11^{a}+15^{a}\right]+ \\
6(n-1) 19^{a}+3(n-2)(n-1)\left[16^{a}+25^{a}\right]+ \\
(6 n-12)\left[24^{a}+29^{a}\right]+3(n-3)(n-2) 34^{a} \\
B_{2}{ }^{a}(T S L(n))=6 \times 12^{a}+(9 n-3)\left[24^{a}+56^{a}\right]+
\end{gathered}
$$

$$
\begin{array}{r}
6(n-1) 84^{a}+3(n-2)(n-1)\left[39^{a}+156^{a}\right]+ \\
(6 n-12)\left[119^{a}+204^{a}\right]+3(n-3)(n-2) 64^{a}
\end{array}
$$

Proof: Let $G$ be the graph of triangular oxide network, $\operatorname{RHSL}(n)$ with

$$
|V(\operatorname{TsL}(n))|=\left[\frac{(n-1)(n-2)}{2}\right] 3(n-1)+n^{2}+3
$$

and

$$
|E(\operatorname{Ts} L(n))|=\frac{3}{2} n(n+1)
$$

(i) We Compute $B_{1}{ }^{a}(T s L(n))$ as follows

$$
\begin{gathered}
B_{1}{ }^{a}(T S L(n))=\sum_{u e}\left[d_{G}(u)+d_{G}(e)\right]^{a} \\
B_{1}^{a}(G)=3\left[7^{a}+7^{a}\right]+(9 n-3)\left[11^{a}+15^{a}\right] \\
+3(n-1)\left[19^{a}+19^{a}\right]+3(n \\
-2)(n-1)\left[16^{a}+25^{a}\right] \\
+(6 n-12)\left[24^{a}+29^{a}\right]+\frac{3}{2}(n-3)(n \\
-2)\left[34^{a}+34^{a}\right] \\
=6 \times 7^{a}+(9 n-3)\left[11^{a}+15^{a}\right]+6(n-1) 19^{a} \\
+3(n-2)(n-1)\left[16^{a}+25^{a}\right] \\
+(6 n-12)\left[24^{a}+29^{a}\right]+3(n-3)(n \\
-2) 34^{a}
\end{gathered}
$$

(ii) We Compute $B_{1}{ }^{a}(\operatorname{Ts} L(n))$ as follows

$$
\begin{aligned}
& B_{2}{ }^{a}(\operatorname{TsL}(n))=\sum_{u e}\left[d_{G}(u) d_{G}(e)\right]^{a} \\
& B_{2}^{a}(G)=3\left[12^{a}+12^{a}\right]+(9 n-3)\left[24^{a}+56^{a}\right] \\
&+3(n-1)\left[84^{a}+84^{a}\right]+3(n \\
&-2)(n-1)\left[39^{a}+156^{a}\right] \\
&+(6 n-12)\left[119^{a}+204^{a}\right] \\
&+ \\
&+\frac{3}{2}(n-3)(n-2)\left[264^{a}+264^{a}\right] \\
& B_{2}^{a}(G)=6 \times 12^{a}+(9 n-3)\left[24^{a}+56^{a}\right]+6(n-1) 84^{a} \\
&+3(n-2)(n-1)\left[39^{a}+156^{a}\right] \\
&+(6 n-12)\left[119^{a}+204^{a}\right]+3(n-3)(n \\
&-2) 64^{a}
\end{aligned}
$$

Corollary: The first and second K-Banhatti indices for a triangular silicate network, $T s L(n)$ are given by

$$
\begin{gathered}
B_{1}(\operatorname{Ts} L(n))=225 n^{2}-213 n+70 \\
B_{2}(\operatorname{Ts} L(n))=1299 n^{2}+1641 n+1416
\end{gathered}
$$

Proof: Putting $a=1$ in equation (7), we get

$$
\begin{gathered}
=42+234 n-78+114 n-114+123 n^{2}-369 n+246 \\
+318 n-636+102 n^{2}-510 n+612 \\
=225 n^{2}-213 n+70
\end{gathered}
$$

Putting $a=1$ in equation (8), we get 


$$
\begin{gathered}
B_{2}=72+720 n-240+504 n-504+507 n^{2}-1521 n+1014+1938 n-3678+792 n^{2}-3960 n+4752 \\
=1299 n^{2}+1641 n+1416
\end{gathered}
$$

Corollary: The first and second K-hyper Banhatti indices for a triangular silicate network $T s L(n)$ are given by

$$
\begin{aligned}
& H B_{1}(\operatorname{Ts} L(n))=11979 n^{2}+46551 n+12222 \\
& H B_{2}(\operatorname{Ts} L(n))=286659 n^{2}-867747 n+685738
\end{aligned}
$$

Proof: Putting $a=2$ in equation (7), we get

$$
\begin{gathered}
=588+6084 n-2028+4332 n-4332+5043 n^{2}-15129 n+10086+16584 n-33708+6936 n^{2}-34680 n+41616 \\
=11979 n^{2}+46551 n+12222
\end{gathered}
$$

Putting $a=2$ in equation (8), we get

$$
\begin{gathered}
=864+33408 n-11136+42336 n-44336+77571 n^{2}-232713 n+155142+334662 n-669324+209088 n^{2} \\
-1045440 n+1254528 \\
=286659 n^{2}-867747 n+685738
\end{gathered}
$$

Corollary: The first and second modified K Banhatti indices for a triangular silicate network $\operatorname{TsL}(n)$ are given by

$$
\begin{gathered}
m B_{1}(\operatorname{Ts} L(n))=3.7058823529411 n^{2}+0.827191373 n+0.299244772 \\
m B_{2}(\operatorname{Ts} L(n))=0.1430288461538 n^{2}+0.5463497899159 n+0.3763938268
\end{gathered}
$$

Proof: Putting $a=-1$ in equation (7), we get

$$
\begin{aligned}
=\frac{6}{7}+(9 n-3)\left(\frac{1}{11}+\frac{1}{25}\right)+ & \frac{6}{19}(n-1)+3(n-2)(n-1)\left(\frac{1}{16}+\frac{1}{25}\right)+(6 n-12)\left(\frac{1}{24}+\frac{1}{29}\right)+\frac{3}{34}(n-3)(n-2) \\
& =3.7058823529411 n^{2}+0.827191373 n+0.299244772
\end{aligned}
$$

Putting $a=-1$ in equation (8), we get

$$
\begin{gathered}
=\frac{6}{12}+(9 n-3)\left(\frac{1}{24}+\frac{1}{56}\right)+\frac{6}{84}(n-1)+3(n-2)(n-1)\left(\frac{1}{39}+\frac{1}{156}\right)+(6 n-12)\left(\frac{1}{119}+\frac{1}{204}\right)+\frac{3}{64}(n-3)(n-2) \\
=0.1430288461538 n^{2}+0.5463497899159 n+0.3763938268
\end{gathered}
$$

\subsection{Calculating Banhatti Indices for Triangular Oxide \\ Network}

A triangular oxide network of dimension $n$ denoted by $\operatorname{TOX}(n)$ is given in figure 2 . The number of vertices and the number of edges in a triangular oxide network are given by

$$
\begin{gathered}
|V(\operatorname{TOX}(n))|=\frac{1}{2}\left(n^{2}-3 n+2\right)+3(n-1)+3 \\
|E(\operatorname{TOX}(n))|=\frac{3}{2} n(n+1)
\end{gathered}
$$

In a triangular oxide network, from level 4 there are four types of edges based on the degree of the vertices of each edge given in table 2 .

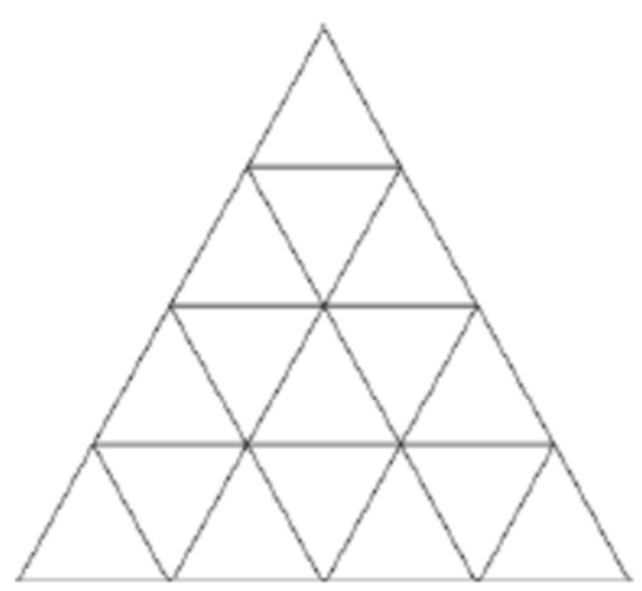

Figure 2. Triangular oxide network TOX(n).

In Table 2 the edge degree partition of triangular oxide network $\operatorname{TOX}(n)$ of order 4 is shown. 
Table 2. Edge degree partition of triangular silicate network TOX $(n)$ of order 4.

\begin{tabular}{lll}
\hline $\boldsymbol{d}_{\boldsymbol{G}}(\boldsymbol{u}), \boldsymbol{d}_{\boldsymbol{G}}(\boldsymbol{e}) \backslash \boldsymbol{e}=\boldsymbol{u} \boldsymbol{v} \in \boldsymbol{E}(\boldsymbol{G})$ & $\boldsymbol{d}_{\boldsymbol{G}}(\boldsymbol{e})$ & Total number of edges \\
\hline$(2,4) 1$ & 4 & 6 \\
$(4,4)$ & 6 & $3(n-1)$ \\
$(4,6) 1$ & 8 & $6(n-2)$ \\
$(6,6) 1$ & 10 & $3 \frac{\left[(n-3)^{2}+(n-3)\right]}{2}$ \\
\hline
\end{tabular}

Theorem: For a triangular oxide network $\operatorname{TOX}(n)$, the general first and second K-Banhatti indices are given by

$$
\begin{array}{r}
B_{1}{ }^{a}(\operatorname{TOX}(n))=6\left(6^{a}+8^{a}\right)+6(n-1) \times 10^{a}+ \\
6(n-2)\left(12^{a}+14^{a}\right)+3\left[(n-3)^{2}+(n-3)\right] \times 16^{a}(17) \\
B_{2}{ }^{a}(\operatorname{TOX}(n))=6\left(8^{a}+16^{a}\right)+6(n-1) \times 24^{a}+ \\
6(n-2)\left(32^{a}+48^{a}\right)+3\left[(n-3)^{2}+(n-3)\right] \times 60^{a}(18)
\end{array}
$$

Proof: Let $G$ be the graph of triangular oxide network, $\operatorname{RHSL}(n) \quad$ with $\quad|V(\operatorname{TOX}(n))|=\frac{1}{2}\left(n^{2}-3 n+2\right)+$ $3(n-1)+3$ and $|E(\operatorname{TOX}(n))|=\frac{3}{2} n(n+1)$.

First we compute the general first K-Banhatti index.

(i) We Compute $B_{1}{ }^{a}(\operatorname{TOX}(n))$ as follows

$$
\begin{gathered}
B_{1}{ }^{a}(\operatorname{TOX}(n))=\sum_{u e}\left[d_{G}(u)+d_{G}(e)\right]^{a} \\
B_{1}^{a}(\operatorname{TOX}(n))=6\left(6^{a}+8^{a}\right)+3(n-1)\left(10^{a}+10^{a}\right) \\
+6(n-2)\left(12^{a}+14^{a}\right) \\
+\frac{3\left[(n-3)^{2}+(n-3)\right]}{2}\left(16^{a}+16^{a}\right) \\
=6\left(6^{a}+8^{a}\right)+ \\
+6(n-1) \times 10^{a}+6(n-2)\left(12^{a}+14^{a}\right) \\
+3\left[(n-3)^{2}+(n-3)\right] \times 16^{a}
\end{gathered}
$$

Now we compute the general second K-Banhatti index.

(ii) We Compute $B_{2}{ }^{a}(T O X(n))$ as follows

$$
\begin{aligned}
B_{2}{ }^{a}( & \text { TOX }(n))=\sum_{u e}\left[d_{G}(u) d_{G}(e)\right]^{a} \\
B_{2}{ }^{a}(\operatorname{TOX}(n)) & =6\left(8^{a}+16^{a}\right)+3(n-1)\left(24^{a}+24^{a}\right) \\
& +6(n-2)\left(32^{a}+48^{a}\right) \\
& +\frac{3\left[(n-3)^{2}+(n-3)\right]}{2}\left(60^{a}+60^{a}\right) \\
=6\left(8^{a}+16\right)+ & 6(n-1) \times 24^{a}+6(n-2)\left(32^{a}+48^{a}\right) \\
& +3\left[(n-3)^{2}+(n-3)\right] \times 60^{a}
\end{aligned}
$$

Corollary: The first and second K-Banhatti indices for a triangular oxide network, $T O L(n)$ are given by

$$
\begin{gathered}
B_{1}(\operatorname{TOX}(n))=48 n^{2}-24 n \\
B_{2}(\operatorname{TOX}(n))=180 n^{2}-276 n+120
\end{gathered}
$$

Proof: Putting $a=1$ in equation (17), we get

$$
\begin{gathered}
=6(6+8)+6(n-1) \times 10+6(n-2)(12+14) \\
+3\left[(n-3)^{2}+(n-3)\right] \times 16 \\
=48 n^{2}-24 n
\end{gathered}
$$

Putting $a=1$ in equation (18), we get

$$
\begin{gathered}
=6(8+16)+6(n-1) \times 24+6(n-2)(32+48) \\
+3\left[(n-3)^{2}+(n-3)\right] \times 60 \\
=180 n^{2}-276 n+120
\end{gathered}
$$

Corollary: The first and second K-hyper Banhatti indices for a oxide network, $\operatorname{TOX}(n)$ are given by

$$
\begin{array}{r}
H B_{1}(\operatorname{TOX}(n))=168 n^{2}-1200 n+528 \\
H B_{2}(\operatorname{TOX}(n))=10800 n^{2}-30576 n+21528
\end{array}
$$

Proof: Putting $a=2$ in equation (17), we get

$$
\begin{gathered}
=6(36+64)+6(n-1) \times 100+6(n-2)(144+196) \\
+3\left[(n-3)^{2}+(n-3)\right] \times 256 \\
=168 n^{2}-1200 n+528
\end{gathered}
$$

Putting $a=2$ in equation (18), we get

$$
\begin{aligned}
=6 & (64+256)+6(n-1) \times 576 \\
& +6(n-2)(1024+2304) \\
& +3\left[(n-3)^{2}+(n-3)\right] \times 3600 \\
& \\
= & 10800 n^{2}-30576 n+21528
\end{aligned}
$$

Corollary: The first and second modified K Banhatti indices for a triangular oxide network, $T O L(n)$ are given by

$$
\begin{gathered}
m B_{1}(\operatorname{TOX}(n))=\frac{3}{16} n^{2}+\frac{331}{560} n+\frac{117}{280} \\
m B_{2}(\operatorname{TOX}(n))=\frac{1}{20} n^{2}+\frac{5}{16} n+\frac{11}{20}
\end{gathered}
$$

Proof: Putting $a=-1$ in equation (17), we get

$$
\begin{aligned}
=6\left(\frac{1}{6}+\frac{1}{8}\right) & +\frac{6}{10}(n-1)+6(n-2)\left(\frac{1}{12}+\frac{1}{14}\right) \\
& +\frac{3}{16}\left[(n-3)^{2}+(n-3)\right] \\
& =\frac{3}{16} n^{2}+\frac{331}{560} n+\frac{117}{280}
\end{aligned}
$$

Putting $a=-1$ in equation (18), we get

$$
\begin{gathered}
=6\left(\frac{1}{8}+\frac{1}{16}\right)+\frac{6}{24}(n-1)+6(n-2)\left(\frac{1}{32}+\frac{1}{48}\right) \\
+\frac{3}{60}\left[(n-3)^{2}+(n-3)\right] \\
=\frac{1}{20} n^{2}+\frac{5}{16} n+\frac{11}{20}
\end{gathered}
$$

\subsection{Calculating Banhatti Indices for Rhombus Silicate Network}

By the different arrangements of the tetrahedron silicate, we can obtain different silicate structures. Similarly, different 
silicate networks are constructed by different silicate structures. The number of vertices and the number of edges in a rhombus silicate network are given by

$$
|V(R H S L(n))|=5 n^{2}+2
$$

and

$$
|E(R H S L(n))|=12 n^{2}
$$

In Figure 3, the rhombus silicate network of dimension 3 is presented.

Table 3. The Edge degree partition of RHSL(n) based on degree of end vertices.

\begin{tabular}{lll}
\hline $\boldsymbol{d}_{\boldsymbol{G}}(\boldsymbol{u}), \boldsymbol{d}_{\boldsymbol{G}}(\boldsymbol{e v})$ & $\boldsymbol{d}_{\boldsymbol{G}}(\boldsymbol{e})$ & $|\boldsymbol{E}(\boldsymbol{R} \boldsymbol{H O X}(\boldsymbol{n}))|$ \\
\hline$(3,3)$ & 4 & $4 n+2$ \\
$(3,6)$ & 7 & $6 n^{2}+4 n-4$ \\
$(6,6)$ & 10 & $\left(6 n^{2}-8 n+2\right) 1$ \\
\hline
\end{tabular}

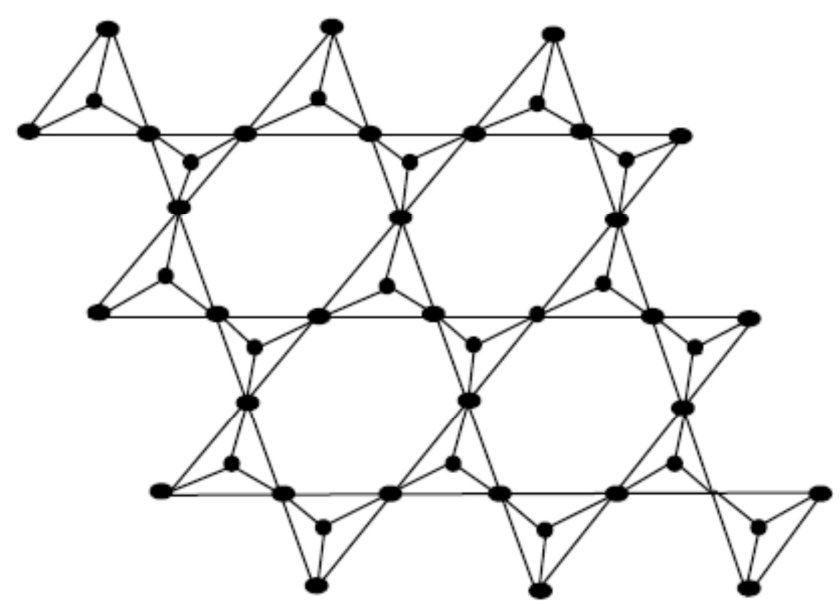

Figure 3. Rhombus silicate $(\operatorname{RHSL}(n))$.

Theorem: For a rhombus silicate network, $\operatorname{RHSL}(n)$ of dimension $n$, the general first and second K-Banhatti indices are given by

$$
\begin{gathered}
B_{1}{ }^{a}(\operatorname{RHSL}(n))=2(4 n+2) 7^{a}+\left(6 n^{2}+4 n-4\right)\left[10^{a}+\right. \\
\left.13^{a}\right]+2\left(6 n^{2}-8 n+2\right) 16^{a} \\
B_{2}{ }^{a}(\operatorname{RHSL}(n))=2(4 n+2) 12^{a}+\left(6 n^{2}+4 n-4\right)\left[21^{a}+\right. \\
\left.42^{a}\right]+2\left(6 n^{2}-8 n+2\right) 60^{a}
\end{gathered}
$$

Proof: Let $G$ be the graph of rhombus silicate network, $\operatorname{RHSL}(n) \quad$ with $\quad|V(\operatorname{RHSL}(n))|=5 n^{2}+2 \quad$ and $|E(R H S L(n))|=12 n^{2}$. There are three types of edges based on degree of end vertices. The edge degree partition of a silicate network SLn is given in Table 3.

To compute $B_{1}{ }^{a}(\operatorname{RHSL}(n))$, we compute the required result as follows:

$$
\begin{gathered}
B_{1}{ }^{a}(\operatorname{RHSL}(n))=\sum_{u e}\left[d_{G}(u)+d_{G}(e)\right]^{a} \\
=(4 n+2)\left[7^{a}+7^{a}\right]+\left(6 n^{2}+4 n-4\right)\left[10^{a}+13^{a}\right] \\
+\left(6 n^{2}-8 n+2\right)\left[16^{a}+16^{a}\right]
\end{gathered}
$$

$$
\begin{gathered}
=2(4 n+2) 7^{a}+\left(6 n^{2}+4 n-4\right)\left[10^{a}+13^{a}\right] \\
+2\left(6 n^{2}-8 n+2\right) 16^{a}
\end{gathered}
$$

To compute $B_{1}{ }^{a}(\operatorname{RHSL}(n))$, we compute the required result as follows:

$$
\begin{gathered}
B_{2}{ }^{a}(\operatorname{RHSL}(n))=\sum_{u e}\left[d_{G}(u) d_{G}(e)\right]^{a} \\
=(4 n+2)\left[12^{a}+12^{a}\right]+\left(6 n^{2}+4 n-4\right)\left[21^{a}+42^{a}\right] \\
+\left(6 n^{2}-8 n+2\right)\left[60^{a}+60^{a}\right] \\
=2(4 n+2) 12^{a}+\left(6 n^{2}+4 n-4\right)\left[21^{a}+42^{a}\right] \\
+2\left(6 n^{2}-8 n+2\right) 60^{a}
\end{gathered}
$$

Corollary: The first and second K-Banhatti indices for a rhombus silicate network, $\operatorname{RHSL}(n)$ are given by

$$
\begin{gathered}
B_{1}(\operatorname{RHSL}(n))=330 n^{2}-108 n \\
B_{2}(\operatorname{RHSL}(n))=1098 n^{2}-612 n+38
\end{gathered}
$$

Proof: Putting $a=1$ in equation (27), we get

$$
\begin{gathered}
=2(4 n+2) 7+\left(6 n^{2}+4 n-4\right)[10+13] \\
+2\left(6 n^{2}-8 n+2\right) 16 \\
=56 n+28+138 n^{2}+92 n-92+192 n^{2}-256 n-108 \\
=330 n^{2}-108 n
\end{gathered}
$$

Putting $a=1$ in equation (28), we get

$$
\begin{aligned}
=2(4 n+ & 2) 12+\left(6 n^{2}+4 n-4\right)[21+42] \\
+ & 2\left(6 n^{2}-8 n+2\right) 60 \\
=96 n+48+ & 378 n^{2}+252 n-252+720 n^{2}-960 n \\
+ & 240 \\
= & 1098 n^{2}-612 n+38
\end{aligned}
$$

Corollary: The first and second K-hyper Banhatti indices for a rhombus silicate network, $R H S L(n)$ are given by

$$
\begin{gathered}
H B_{1}(R H S L(n))=4506 n^{2}-2748 n+264 \\
H B_{2}(R H S L(n))=56430 n^{2}-47628 n+6156
\end{gathered}
$$

Proof: Putting $a=2$ in equation (27), we get

$$
\begin{array}{rl}
=98(4 n+2)+ & 239\left(6 n^{2}+4 n-4\right)+512\left(6 n^{2}-8 n+2\right) \\
=392 n+1 & 96+1434 n^{2}+956 n-956+3072 n^{2} \\
- & 4096 n+1024 \\
= & 4506 n^{2}-2748 n+264
\end{array}
$$

Putting $a=2$ in equation (28), we get

$$
\begin{aligned}
=288 & (4 n+2)+2205\left(6 n^{2}+4 n-4\right) \\
& +7200\left(6 n^{2}-8 n+2\right) \\
=1152 n+576 & +13230 n^{2}+8820 n-8820+43200 n^{2} \\
& -57600 n+14400
\end{aligned}
$$




$$
=56430 n^{2}-47628 n+6156
$$

Corollary: The first and second modified K Banhatti indices for a rhombus silicate network, $\operatorname{RHSL}(n)$ are given by

$$
\begin{gathered}
=\frac{2}{7}(4 n+2)+\left(6 n^{2}+4 n-4\right)\left[\frac{1}{10}+\frac{1}{13}\right]+\frac{2}{16}\left(6 n^{2}-8 n+2\right) \\
=\frac{2}{7}(4 n+2)+\frac{23}{130}\left(6 n^{2}+4 n-4\right)+\frac{1}{8}\left(6 n^{2}-8 n+2\right) \\
=\frac{8}{7} n+\frac{4}{7}+\frac{69}{65} n^{2}+\frac{46}{65} n-\frac{46}{65}-\frac{3}{4} n^{2}-n+\frac{1}{4}
\end{gathered}
$$

Putting $a=-1$ in equation (28), we get$$
=\frac{2}{12}(4 n+2)+\left(6 n^{2}+4 n-4\right)\left[\frac{1}{21}+\frac{1}{42}\right]+\frac{2}{602}\left(6 n^{2}-8 n+2\right)
$$$$
=\frac{3}{2} n+\frac{1}{3}+\frac{3}{7} n^{2}+\frac{2}{7} n-\frac{2}{7}+\frac{1}{5} n^{2}-\frac{4}{15} n+\frac{1}{15}
$$

\subsection{Calculating Banhatti Indices for Rhombus Oxide Network}

If we delete all the silicon ions from the rhombus silicate network, then we obtain rhombus oxide network as shown in Figure 4 of dimension 3 .

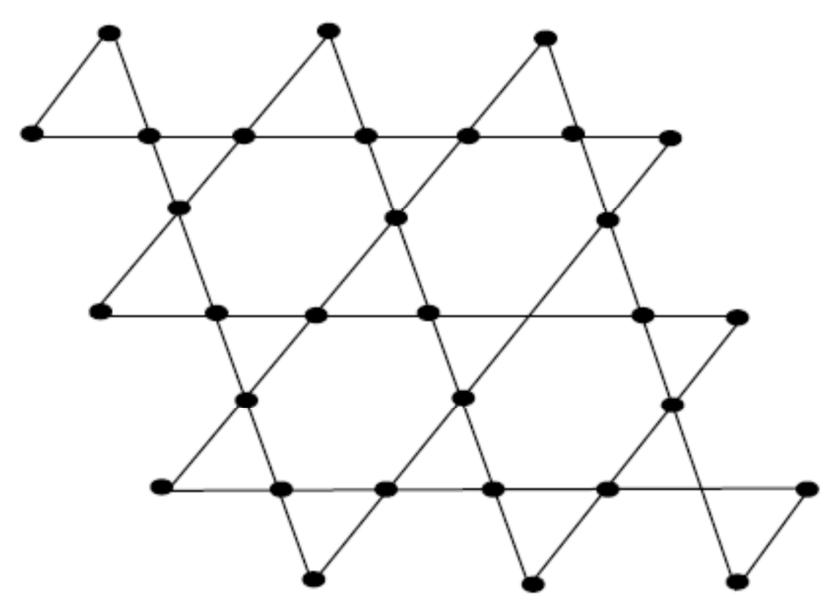

Figure 4. Rhombus oxide network, RHOX(n) of dimension $n$.

The number of vertices and the number of edges in a rhombus oxide network are given by

$$
|V(R H O X(n))|=3 n^{2}+2 n
$$

and

$$
|E(R H O X(n))|=6 n^{2}
$$

Theorem: For a rhombus oxide network, $\operatorname{RHOX}(n)$ of dimension $n$, the general first and second K-Banhatti indices are given by

$$
\begin{aligned}
& B_{1}{ }^{a}(R \operatorname{RHOX}(n))= 4 \times 4^{a}+4(2 n-1)\left[6^{a}+8^{a}\right]+ \\
& 2\left(6 n^{2}-8 n+2\right) 10^{a}
\end{aligned}
$$

$$
\begin{aligned}
B_{2}{ }^{a}(\operatorname{RHOX}(n))= & 4 \times 4^{a}+4(2 n-1)\left[8^{a}+16^{a}\right]+ \\
& 2\left(6 n^{2}-8 n+2\right) 24^{a}
\end{aligned}
$$

Proof: Let $G$ be the graph of rhombus oxide network with $|V(R H O X(n))|=3 n^{2}+2 n$ and $|E(R H O X(n))|=6 n^{2}$. There are three types of edges based on degree of end vertices. The edge degree partition of the rhombus oxide network, $\operatorname{RHOX}(n)$ is given in Table 4.

Table 4. The Edge degree partition of RHOX $(n)$ based on degree of end vertices.

\begin{tabular}{lll}
\hline $\boldsymbol{d}_{G}(\boldsymbol{u}), \boldsymbol{d}_{G}(\boldsymbol{e v})$ & $\boldsymbol{d}_{G}(\boldsymbol{e})$ & $|\boldsymbol{E}(\boldsymbol{R H O X}(\boldsymbol{n}))|$ \\
\hline$(2,2)$ & 2 & 2 \\
$(2,4)$ & 4 & $4(2 n-1)$ \\
$(4,4) 1$ & 6 & $\left(6 n^{2}-8 n+2\right)$ \\
\hline
\end{tabular}

(i) To compute $B_{1}{ }^{a}(R H O X(n))$, we compute the required result as follows:

$$
\begin{gathered}
B_{1}{ }^{a}(\operatorname{RHOX}(n))=\sum_{u e}\left[d_{G}(u)+d_{G}(e)\right]^{a} \\
=2\left[4^{a}+4^{a}\right]+4(2 n-1)\left[6^{a}+8^{a}\right] \\
+\left(6 n^{2}-8 n+2\right)\left[10^{a}+10^{a}\right]
\end{gathered}
$$

$=4 \times 4^{a}+4(2 n-1)\left[6^{a}+8^{a}\right]+2\left(6 n^{2}-8 n+2\right) 10^{a}$

(ii) To compute $B_{2}{ }^{a}(R H O X(n))$, we compute the required result as follows:

$$
\begin{gathered}
B_{2}{ }^{a}(\operatorname{RHOX}(n))=\sum_{u e}\left[d_{G}(u) d_{G}(e)\right]^{a} \\
B_{2}{ }^{a}(\operatorname{RHOX}(n))=2\left[4^{a}+4^{a}\right]+4(2 n-1)\left[8^{a}+16\right] \\
+\left(6 n^{2}-8 n+2\right)\left[24^{a}+24^{a}\right] \\
=4 \times 4^{a}+4(2 n-1)\left[8^{a}+16^{a}\right]+2\left(6 n^{2}-8 n+2\right) 24^{a}
\end{gathered}
$$

Corollary: The first and second K-Banhatti indices for a rhombus oxide network, $\operatorname{RHOX}(n)$ are given by 


$$
\begin{gathered}
B_{1}(\operatorname{RHOX}(n))=120 n^{2}-48 n \\
B_{2}(R \operatorname{HOX}(n))=288 n^{2}-192 n+16
\end{gathered}
$$

Proof: Putting $a=1$ in equation (37), we get

$$
\begin{gathered}
=4 \times 4+4(2 n-1)[6+8]+2\left(6 n^{2}-8 n+2\right) 10 \\
=16+112 n-56+120 n^{2}-160 n+40 \\
=120 n^{2}-48 n
\end{gathered}
$$

Putting $a=1$ in equation (38), we get

$$
\begin{gathered}
=4 \times 4+4(2 n-1)[8+16]+2\left(6 n^{2}-8 n+2\right) 24 \\
=16+192 n-96+288 n^{2}-384 n+96 \\
=288 n^{2}-192 n+16
\end{gathered}
$$

Corollary: The first and second K-hyper Banhatti indices for a rhombus oxide network, $R H O X(n)$ are given by

$$
\begin{gathered}
H B_{1}(R H O X(n))=1200 n^{2}+800 n+64 \\
H B_{2}(R H O X(n))=6912 n^{2}-6656 n-192
\end{gathered}
$$

Proof: Putting $a=2$ in equation (37), we get

$$
\begin{gathered}
=4 \times 4^{2}+4(2 n-1)\left[6^{2}+8^{2}\right]+2\left(6 n^{2}-8 n+2\right) 10^{2} \\
=64+4(2 n-1)[100]+200\left(6 n^{2}-8 n+2\right) \\
=64+800 n-400+1200 n^{2}-1600 n+400 \\
=1200 n^{2}+800 n+64
\end{gathered}
$$

Putting $a=2$ in equation (38), we get

$$
\begin{gathered}
=4 \times 4^{2}+4(2 n-1)\left[8^{2}+16^{2}\right]+2\left(6 n^{2}-8 n+2\right) 24^{2} \\
=64+2560 n-2560+6912 n^{2}-9216 n+2304 \\
=6912 n^{2}-6656 n-192
\end{gathered}
$$

Corollary: The first and second modified $\mathrm{K}$ Banhatti indices for a rhombus oxide network, $\operatorname{RHOX}(n)$ are given by

$$
\begin{array}{r}
m B_{1}(R H O X(n))=\frac{5}{6} n^{2}-48 n \\
m B_{2}(R H O X(n))=288 n^{2}-192 n+16
\end{array}
$$

Proof: Putting $a=-1$ in equation (37), we get

$$
\begin{gathered}
=1+4(2 n-1)\left[\frac{1}{6}+\frac{1}{8}\right]+\frac{1}{5}\left(6 n^{2}-8 n+2\right) \\
=1+(2 n-1) \frac{7}{6}+\frac{1}{5}\left(6 n^{2}-8 n+2\right) \\
=\frac{6}{5} n^{2}+\frac{3}{5} n+\frac{7}{30}
\end{gathered}
$$

Putting $a=-1$ in equation (38), we get

$$
=1+4(2 n-1)\left[\frac{1}{8}+\frac{1}{16}\right]+\frac{1}{12}\left(6 n^{2}-8 n+2\right)
$$

$$
\begin{gathered}
=1+\frac{3}{4}(2 n-1)+\frac{1}{12}\left(6 n^{2}-8 n+2\right) \\
=288 n^{2}-192 n+16
\end{gathered}
$$

\section{Conclusion}

In this paper we have computed the exact values of the general K-Banhatti indices, first and second K-Banhatti indices, $\mathrm{K}$ hyper Banhatti indices and modified $\mathrm{K}$ Banhatti indices for triangular silicate network, triangular oxide network, rhombus oxide network and rhombus silicate networks that will help to understand the physical features, chemical reactivities and biological activities of the triangular silicate network, triangular oxide network, rhombus oxide network and rhombus silicate networks. These results can also provide a significant determination in the pharmaceutical industry.

\section{Acknowledgements}

The authors are very grateful to the editor and reviewers for their comments and suggestions, which are helpful in improving the paper.

\section{References}

[1] V. R. Kulli, K-Banhatti indices of graphs, Journal of Computer and Mathematical Sciences, 7(4) (2016) 213-218.

[2] V. R. Kulli, On K Banhatti indices and K hyper-Banhatti indices of V-Phenylenic nanotubes and nanotorus, Journal of Computer and Mathematical Sciences, 7(6) (2016) 302-307.

[3] Gutman, V. R. Kulli, B. Chaluvaraju and H. S. Baregowda, On Banhatti and Zagreb indices, Journal of the International Mathematical Virtual Institute, 7(2017) 53-67.

[4] V. R. Kulli, B. Chaluvaraju and H. S. Boregowda, Connectivity Banhatti indices for certain families of benzenoid systems, Journal of Ultra Chemistry, 13(4) (2017) 81-87.V. R. Kulli, Multiplicative hyper-Banhatti indices and coindices of graphs, International Journal of Mathematical Archive, 7(6) (2016) 60-65.

[6] V. R. Kulli, A new Banhatti geometric-arithmetic index, International Journal of Mathematical Archive, 8(4) (2017) $112-115$.

[7] Wiener, H. Structural determination of paraffin boiling points. J. Am. Chem. Soc. 1947, 69, 17-20.

[8] V. R. Kulli Computing Banhatti Indices of Networks, International Journal of Advances in Mathematics Volume 2018, Number 1, Pages 31-40, 2018.

[9] Garcia, I.; Fall, Y.; Gomez, G. Using topological indices to predict anti-alzheimer and antiparasitic GSK-3 inhibitors by multi-target QSAR in silico screening. Molecules 2010, 15, 5408-5422.

[10] Furtula, B.; Gutman, I.; Dehmer, M. On structure-sensitivity of degree based topological indices. Appl. Math. Comput. 2013, 219, 8973-8978. 
[11] Hao, J. Theorems about Zagreb Indices and Modified Zagreb Indices. MATCH Commun. Math. Comput. Chem. 2011, 65, 659-670.

[12] Xu, K.; Liu, M.; Das, K. C.; Gutman, I.; Furtula, B. A survey on graphs extremal with respect to distance-based topological indices. MATCH Commun. Math. Comput. Chem. 2014, 71, 461-508.

[13] Klavzar, S.; Deutsch, E. M-Polynomial and Degree-Based Topological Indices. Iran. J. Math. Chem. 2015, 6, 93-102.
[14] Dobrynin, A. A.; Entringer, R.; Gutman, I. Wiener index of trees: Theory and applications. Acta Appl. Math. 2001, 66, 211-249.

[15] Zhong, L.: The harmonic index for graphs. Appl. Math. Lett. 2012, 25, 561-566.

[16] Gutman, I. Degree-Based topological indices. Croat. Chem. Acta 2013, 86, 315-361. 